\title{
Das Cholesterin und seine Begleitsubstanzen im menschllchen Depotfett beim Carcinom.
}

\author{
Von \\ Dr. Leonhard Wacker, Ghemiker. \\ Mit drei Kurven im Text.
}

(Aus dem pathologischen Institut der Universität München. Direktor: Professor Borst.) (Der Redaktion zugegangen am 23. Juli 1912.)

Die Untersuchung des menschlichen Depotfettes beim Krebs ${ }^{1}$ ) hat bezüglich seiner chemischen Zusammensetzung keine Eigentümlichkeiten ergeben. Dagegen unterscheidet es sich vom normalen Menschenfett durch eine Vermehrung der unverseifbaren Bestandteile, d. h. durch eine bedeutende Zunahme des Gehaltes der auch unter physiologischen Verhältnissen vorhandenen, in demselben gelösten Cholesterinsubstanzen.

Verseift man Fett mit Kalilauge, so läßt sich aus dem verseiften Produkte mit Schwefeläther eine myelinbildende Substanz, *das Unverseifbare», extrahieren. Es besteht etwa zu einem Dritteil aus dem leicht krystallisierbaren, und dadurch sofort in die Augen fallenden Cholesterin, zu zwei Dritteilen aber aus einem bei 25 bis 32 Grad Celsius schmelzenden Begleitprodukt von wachsartiger Konsistenz, das vielleicht auch aus einem hochmolekularen Alkohol oder Ester desselben besteht. Es soll in der Folge als «Begleitsubstanz» bezeichnet werden. Diese Substanz erwies sich als asche-, stickstoff- und phosphorfrei, löst Cholesterin leicht auf und verhindert dessen Krystallisation.

Die chemische Bindungsweise dieser beiden Stoffe im Fette selbst ist vorläufig unbekannt und geht aus meinen Analysen nicht hervor, denn durch den Isolierungsprozeß nach vorangegangener Verseifung des großen Fettüberschusses können

1) Diese Zeitschrift, Bd. 78 (1912), S. 349.

Hoppe-Seyler's Zeitschrift f. physiol. Chemie. LXXX. 
diese Substanzen natürlich auch hydrolytisch gespalten worden sein. Allenfalls vorhandener Cholesterinfettsäure-Ester würde demnach in Seife und freies Cholesterin zerlegt worden sein.

Die Bezeichnung «Unverseifbares» ist daher unkorrekt, denn gerade das Cholesterin ist vielfach als Ester vorhanden, es sei nur an die Cholesterin-Ester-Verfettung (Cholesterinsteatose) erinnert. Um neue Namen zu vermeiden, will ich jedoch die althergebrachte Bezeichnung beibehalten.

Das Unverseifbare enthält ferner sehr geringe Mengen eines in kaltem Alkohol schwerlöslichen, bei $62-63^{\circ}$ schmelzenden Körpers, über dessen chemische Natur und physiologische Bestimmung einstweilen nichts ermittelt werden konnte.

Neben dem Unverseifbaren befindet sich im Depotfett auch ein Phosphatid, vielleicht in Form von Lecithin. Eine Isolierung dieser Substanz ist der geringen Menge wegen, im Maximum 0,07 Prozent, nicht möglich. In manchen Fetten waren nur Spuren zu finden, beim Carcinom scheint das Phosphatid etwas reichlicher vorhanden zu sein, doch konnte ich eine Gesetzmäßigkeit einstweilen nicht erkennen. Zum Vergleiche herangezogenes Kuhbutterfett war immer phosphorhaltig.

Ich habe in einer größeren Anzahl von Fällen den Gehalt an unverseifbaren Substanzen im Fette Carcinomatöser mit demjenigen des Fettes unter anderen pathologischen, sowie physiologischen Verhältnissen verglichen und eine Vermehrung um das zwei- bis dreifache gegenüber der Norm gefunden. Allein diese Erscheinung findet sich auch bei Tuberkulose, chronischer Sepsis, Diabetes usw., ist also für Carcinom nicht spezifisch. Diese Befunde decken sich im wesentlichen mit denjenigen von Peirce, ${ }^{1}$ ) welcher in der Galle bei gleichen oder ähnlichen Todesursachen ebenfalls eine Erhöhung des Cholesteringehaltes nachweisen konnte. Das Cholesterin befand sich in der Galle größtenteils als freies Cholesterin, während es im Blutplasma meist als Ester vorhanden ist. ${ }^{2}$ )

1) Peirce, Archiv für klinische Medizin, Bd. 106, S. 337.

2) K. Hürthle, Über die Fettsäurecholesterinester des Serums. Diese Zeitschrift, Bd. 21, S. 333, 1895/96. 
Wie schon im ersten Teil dieser Untersuchung (loc. cit.) erwähnt, sind Depotfette mit hohem Gehalt an Unverseif barem sehr häufig, wenn auch nicht ausnahmslos, durch eine intensiv gelbe Farbe gekennzeichnet. Eine Anreicherung solcher cholesterinreicher Lipoidsubstanzen macht sich allenthalben durch die gelben Begleitsubstanzen «die Lipochrome» bemerkbar. Es sei nur an die gelbe Farbe der Nebennieren, der Xanthome und der Hühnerleber im Embryonalstadium erinnert. ${ }^{1}$ )

Die Erhöhung des Unverseifbaren beim Carcinom macht sich in der Regel zuerst beim Mesenterialfett bemerkbar, das schon unter physiologischen Verhältnissen gegenüber dem Unterhautfett einen Mehrgehalt zeigt. Erst in vorgeschrittenen Stadien scheint sich das subcutane Fett mehr und mehr zu beladen. Das Mesenterialfett besitzt auch meist eine gelbere Farbe als das Unterhautfett.

Diese Ansammlung von Cholesterin und seiner Begleitsubstanzen gibt $z u$ folgenden Fragestellungen Anlaß :

1. Kann die Vermehrung ganz oder teilweise auf Zellzerfall zurückgeführt werden?

2. Ist die Erhöhung durch Einschmelzung von Neutralfett unter Retention der Cholesterinsubstanzen erklärbar?

3. Sind Anzeichen vorhanden, daß diese Anreicherung als eine Abwehrmaßregel oder Entgiftungserscheinung des Organismus aufzufassen ist?

Die Beantwortung dieser Fragen ist keineswegs leicht und wir kommen dabei über Vermutungen kaum hinaus. Immerhin bestehen verschiedene Möglichkeiten, die zu erörtern sind:

Es ist naheliegend, die Ursache der Anhäufung in dem bekannten destruktiven Charakter der Geschwülste zu suchen. Die negative Stickstoff bilanz der Krebskranken und der Krankheitsverlauf selbst lehren, daß Zellen zerfallen und demnach

1) Hanes, Über das Vorkommen und die Bedeutung von aniso-n tropen Lipoiden in der Leber des Hühnerembryos. Centralblatt für Pathologie und pathologische Anatomie, Bd. 23 (1912), S. 533. 
Cholesterin in Freiheit gesetzt werden muß. 1) Da mit dem Cholesterin gleichzeitig dessen «Begleitsubstanz» vermehrt wird, so ginge daraus hervor, da $ß$ auch diese durch Zellzerfall entsteht.

Einige weiter zu besprechende Erscheinungen lassen aber doch Zweifel aufkommen, ob das Gesagte unbedingt richtig ist. Auch existiert noch eine andere Möglichkeit der Cholesterinanhäufung.

Durch Einschmelzung von Fett bei diesen konsumierenden Krankheiten wie Krebs, Tuberkulose, chron. Sepsis, könnte lediglich das Neutralfett dem Depot als Energiespender entnommen werden, während die in demselben gelösten Bestandteile zurückbleiben. Auf diese Weise würde eine konzentriertere Lösung (atrophisches Fett) entstehen. Gegen diese Anschauung spricht, daß bei Carcinom, Tuberkulose usw. nach Peirce (loc. cit.) mehr Cholesterin durch die Galle abgeführt wird als unter normalen Verhältnissen. Auch das Auftreten der Erhöhung des Unverseifbaren bei Carcinomfällen, die laut Sektions-Protokoll bei gutem Ernährungszustand verliefen bezw. von Adipositas universalis (Nr. 9, 13 u. 20 Taf. Ia) begleitet waren, lehrt das Gegenteil.

Bemerkenswert ist, daß bei einem ulcerierten Sarkom (Nr. 22, Taf. I) die Menge des Unverseifbaren nicht in dem Maße erhöht war wie beim Carcinom, obwohl beim Sarkom doch sicher auch viele Zellen zerfallen. Bei Pneumonie (Nr. 15, Taf. I) werden auch die Blutzellen zerstört, ohne daß sich dies im Fette bemerkbar macht. Dagegen zeigt sich bei Embolie nach Exstirpation des 0variums wegen eines multilokulären serösen Kystoms, das nicht etwa carcinomatös entartet war, (Nr. 32, Taf. I), besonders im Mesenterialfett eine sehr reichliche Vermehrung der Cholesterinsubstanzen. Die Zunahme beim Diabetes (Nr. 30, Taf. I) könnte mit derselben Wahrscheinlichkeit auf cholesterinreiche Fleischnahrung als auf Zellzerfall zurück-

1) Es ist eine bekannte Erscheinung, daß bei alten Leuten und alten Tieren das Fett besonders stark pigmentiert ist. Auch hier ist die gelbe Farbe auf einen erhöhten Gehalt an Cholesterinsubstanz zurückzuführen. Diese Zunahme findet ihre Erklärung vielleicht auch durch Marasmus und Zellzerfall. 
geführt werden. Es liegen demnach unklare Verhältnisse vor, doch ist es nach dem früher Gesagten wahrscheinlich, daß der Cholesterinüberschuß wenigstens teilweise vom Zellzerfall stammt.

Die Lipoidämie beim Coma diabeticum, ${ }^{1}$ ) ferner die Beobachtungen von Albrecht und Weltmann') und von W. Hueck, ${ }^{3}$ ) wonach bei Carcinom, Tuberkulose u. s. w. die doppelt lichtbrechende Substanz, d. h. der Cholesterin-Ester, sowie das Cholesterin überhaupt, in den Nebennieren stark verringert ist, oder ganz fehlt, sprechen für die Ansicht, daß das Cholesterin bei einem aktiven Vorgange beteiligt ist, denn anders kann man sich die Tatsache nicht erklären, daß gerade jenes Organ, welches sonst das Cholesterin-Depot zu sein scheint, bei einer Überschwemmung des Körpers mit dieser Substanz ganz frei davon bleiben sollte; und dies berechtigt uns zu der Fragestellung:

Ist diese Anreicherung als eine Abwehrmaßregel oder Entgiftungerscheinung des Organismus aufzufassen?

Ein anderes Beispiel aus der Pathologie wird uns der Beantwortung dieser Frage vielleicht näher bringen:

Während das Cholesterin als Bestandteil der Zellhüllen wohl meist als freies Cholesterin vorzukommen pflegt, finden wir es bei pathologischen Prozessen sowohl als auch in den Nebennieren in Form von doppeltbrechenden Fettsäureestern. So hat z. B. Windaus ${ }^{4}$ ) in der Amyloid-Niere und in der atheromatösen Aorta eine Vermehrung des freien Cholesterins um das 6 fache, des Esters. dagegen um das 20 fache ermittelt. Das Verhältnis des freien Cholesterins zum Ester verschiebt sich zugunsten des letzteren und stellt nicht bloß ein Multiplum des Verhältnisses in der normalen Aorta dar. Es ist also dabei ein chemischer Vorgang im Spiele. Die Bildung von doppeltbrechenden Cholesterinestern setzt voraus, daß freie

1) Vgl. v. Noorden, .Die Zuckerkrankheit», Berlin 1910, S. 151.

2) Wiener med. Wochenschrift 1911, Nr. 14.

8) Werner Hueck, Sitzung des ärztlichen Vereins München. Münchener med. Wochenschrift 1911, S. 2588.

4) Diese Zeitschrift, Bd. 65 (1910), S. 110; Bd. 67 (1910) S. 175. 
Säure und freies Cholesterin vorhanden gewesen sein müssen. Wenn also in einem Gewebe doppelt-lichtbrechende Substanz sichtbar wird, so hat vorher eine Säurebildung stattgefunden. Die Zunahme des freien Cholesterins in der atheromatösen Aorta erklärt sich in ungezwungener Weise durch die Eigenschaft des Cholesterins, mit Säuren lockere Verbindungen einzugehen, die bei der Analyse als freies Cholesterin erscheinen. ${ }^{1}$ ) A. v. Baeyer und Victor Villiger ${ }^{2}$ ) haben vor längerer Zeit auf die basischen Eigenschaften des Sauerstoffs hingewiesen. Auch das Sauerst of fat om im Gholesterin besitzt schwach basische Eigenschaften, es zählt daher das Cholesterin auch zu jenen Verbindungen, welche Oxoniumsalze bilden. Die von den genannten Autoren in den Berichten 34, S. 2691 aufgeführten Beispiele für solche Sauerstoff basen wie Borneol, Menthol und Tetrahydrocarveol sind gleich dem Cholesterin als sekundäre Ringalkohole aufzufassen und gehören sogar zu ähnlichen Körperklassen.

Diese basischen Eigenschaften könnten als die Ursache angesehen werden, weshalb das Cholesterin in Form locker gebundener Sauerstoffsalze mit den Fettsäuren in der atheromatösen Aorta festgehalten wird, um später unter Wasserabspaltung in den stabilen Ester ${ }^{3}$ ) überzugehen.

Ähnlich könnte man beim Carcinom an eine Entsäuerung des Organismus durch das Cholesterin denken, denn durch Zerfall und Oxydation von phosphor- und schwefelhaltigen Eiweißkörpern entsteht ja auch hier Säure.

1) Biochemisches Handlexikon, Bd. 3, S. 273. - Graven Moore, Medical Chronicle, Dez. 1907. - P. White, Medical Chronicle March. 1908. Proceedings of the Physio. Soc., Dec. 1908.

$\left.{ }^{2}\right)$ A. v. Baeyer und Viktor Villiger, Ber. d. deutsch. chem. Ges., Bd. 34, 1901, S. 2679 und 3612; Bd. 35 (1902), S. 1201.

3) Aucb unter physiologischen Verhältnissen scheint dem Cholesterin eine fettsäurebindende Rolle zuzukommen. Hanes (loc. cit.) nimmt, auf Grund mikroskopischer Untersuchungen an der Leber des Hühnerembryos, an, daß die Knochenbildung, d. h. die Umwandlung des organischgebundenen Phosphors in die anorganische Form bei Vögeln (wie Säugetieren) unter Abspaltung von Fettsäure sich vollzieht, welch letztere mit Cholesterin gekuppelt, im Mikroskope als anisotrope Substanz Cholesterinfettsäureester - erscheint. 
Die andere Art der Schutzwirkung des Cholesterins kann bekanntlich darin bestehen, daß es mit schädlichen Substanzen, ähnlich dem Digitonin oder Sapotoxin, unlösliche Doppelverbindungen gibt und dadurch die Giftwirkung aufhebt.

Auf zweierlei Weise könnten also die Cholesterinsubstanzen beim Carcinom oder anderen Krankheiten den Organismus vor Schädigungen schützen, woraus sich das Fehlen derselben in den Nebennieren erklären ließe.

\section{Experimenteller Teil.}

Das menschliche Depotfett desselben Individuums weist schon unter physiologischen Verhältnissen Schwankungen in seinem Gehalte an unverseifbarer Substanz auf. Für vergleichende Untersuchungen über den Gehalt an solchen hochmolekularen Alkoholen empfiehlt es sich daher, nur Fette von dem gleichen Depot zu wählen. Mesenterialfett oder Unterhautfett des Oberschenkels sind besonders geeignet, weil sie meist in reichlicher Menge zur Verfügung stehen.

Zur Fettgewinnung wird das zerkleinerte Material in einer Schale am Wasserbad ausgeschmolzen und filtriert. Es ist zweckmäßig, die Filtration erst dann vorzunehmen, wenn sich eine reichliche Fettmenge angesammelt hat, weil das ausgeschmolzene Fett durch Wärmeleitung den Prozeß beschleunigt.

Das Mesenterialfett ist vielfach wasserhaltig und muß im vorgewärmten Scheidetrichter vom Wasser getrennt und nochmals in der Schale am Wasserbad getrocknet werden.

Im Butterrefraktometer zeigen die gelben, cholesterinreichen Fette einen blaugrünen bis gelbgrünen Rand, während die normalen Fette einen blauen Dispersionsrand besitzen (Unterhautfette Neugeborener mit niedriger Refraktometerzahl zeigen gar keinen gefärbten Rand). Ferner haben die lipoidreichen Fette eine verhältnismäßig niedrige, der Jodzahl nicht entsprechende Verseifungszahl. Das Vorhandensein hochmolekularer Alkohole in einem Fette muß natürlich die Menge des zur Verseifung erforderlichen Alkalis, d. h. die Verseifungszahl herunterdrücken.

Zur Bestimmung der unverseif baren Substanz wurden $50 \mathrm{~g}$ 
Fett nach der Methode von Bömer ${ }^{1}$ ) mit alkoholischer Kalilauge verseift und nach dem Verdünnen mit Wasser dreimal mit Äther extrahiert. Der Ätherrückstand wurde ein zweites Mal mit alkoholischer Kalilauge aufgekocht und wiederum mit Äther extrahiert. Nach dreimaligem Waschen des Äthers mit Wasser zur Entfernung der Seife destilliert man den Äther in einem vorher gewogenen Kolben ab und trocknet bei 100 bis $110^{\circ}$. Man erhält so eine mehr oder weniger gelb gefärbte amorphe Substanz von wachsartiger Konsistenz und spezifischem Geruch, die bei anhaltendem Trocknen (2-3 Stunden) eine hellere Farbe annimmt. Sie besitzt die Eigenschaft, in Berührung mit Wasser aufzuquellen, dasselbe in sich aufzunehmen und zeigt unter dem Mikroskop Myelinfiguren.

Zur Bestimmung des Cholesteringehaltes in diesem unverseifbaren Rückstand nach Windaus, loc. cit., löst man letzteren in etwa der achtfachen, dem Volumen nach genau bekannten Menge Alkohol und versetzt $5 \mathrm{ccm}$ davon mit 10-15 ccm einer $1 \%$ igen Digitoninlösung in $90 \%$ igem Alkohol und verfährt im übrigen nach Vorschrift.

Zwischen dem mittleren Cholesteringehalt des Unverseifbaren bei «Carcinom und Nichtcarcinom * besteht nur ein sehr geringer Unterschied, denn das Unverseifbare des Mesenterialfettes beim Carcinom (Taf. 3 a) enthält 38,6\% Cholesterin gegenüber 38,7\% bei Nichtcarcinom(Taf. 3). Ähnlich liegen die Verhältnisse beim Unterhautfett. Aus Taf. 2 und 2 a geht hervor, daß der Cholesteringehalt beim Carcinom 34,9, beim Nichtcarcinom $33,5 \%$ beträgt. Größer sind die Schwankungen dagegen beim Unterhautfett und Mesenterialfett im allgemeinen. Das Unverseifbare des Unterhautfettes zeigte im Mittel aus 41 Fällen (Taf. 2 und 2 a) $34,2 \%$ Cholesterin, während das Mesenterialfett 4,5\% mehr, d. h. 38,7\% (Mittel aus 37 Fällen, Taf. 3 und 3 a) ergab.

Die nachfolgenden Tabellen und graphischen Darstellungen machen die Menge des Unverseifbaren und des Cholesterins bei verschiedenen Krankheiten, verglichen mit Carcinom, ersichtlich.

1) Bömer, Zeitschrift für Untersuchung der Nahrungs- und Genußmittel 1898, S. 38, sowie die Lehrbücher zur Nahrungsmittelchemie. 
Das Cholesterin und seine Begleitsubstanzen.

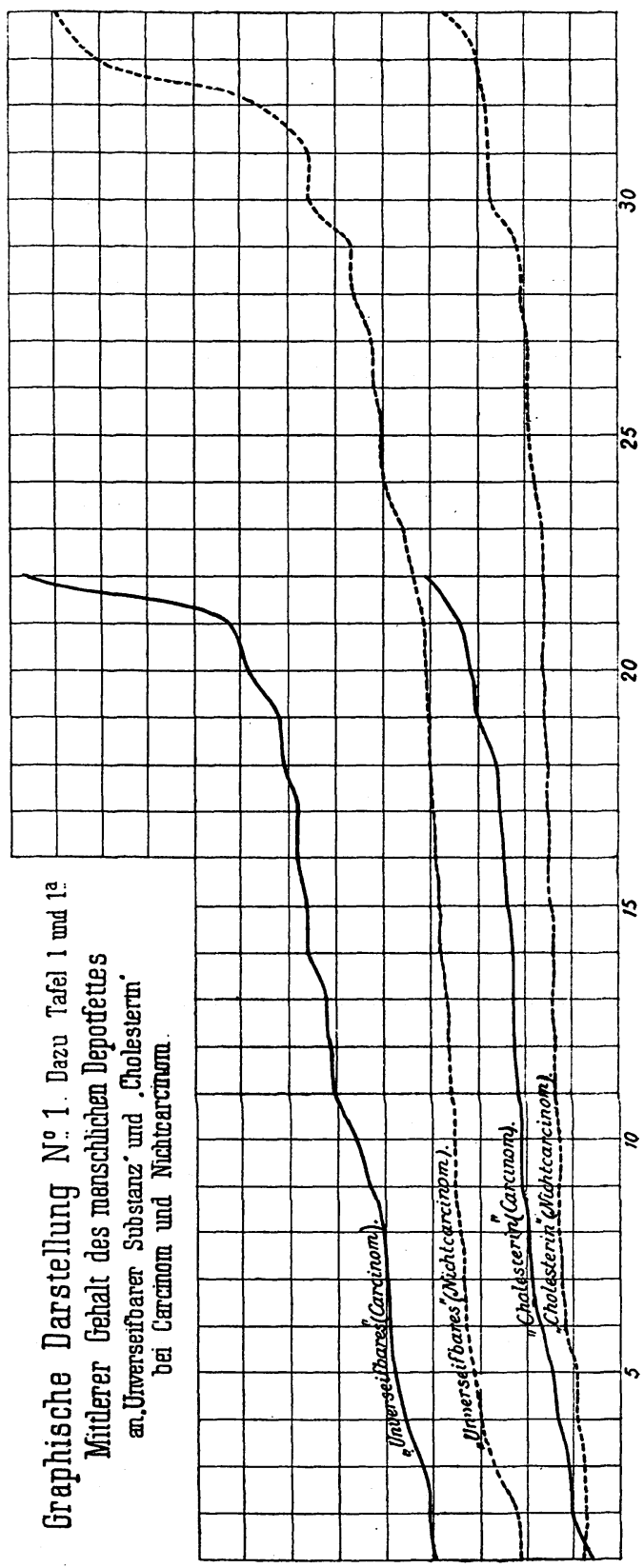


Tafel I. Gehalt des menschlichen Depotfettes an $\mathrm{Zu}$ graphischer

\begin{tabular}{|c|c|c|c|c|c|}
\hline $\mathrm{Nr}$. & $\begin{array}{l}\text { Sektion } \\
\left.\text { Nr. }{ }^{1}\right)\end{array}$ & Diagnose & $\begin{array}{c}\text { Ge- } \\
\text { schlecht } \\
\text { und } \\
\text { Alter }\end{array}$ & $\mid \begin{array}{c}\text { Refrakto- } \\
\text { meter- } \\
\text { zahl } \\
\text { bei } 40^{\circ} \mathrm{C} .\end{array}$ & $\begin{array}{c}\text { Farbe des } \\
\text { Refrakto- } \\
\text { meterrandes }\end{array}$ \\
\hline & - & - & - & 47,6 & schwach blau \\
\hline 1 & 6 & Bronchopneumonie & t $11 / 4$ & 49,4 & blau \\
\hline 2 & - & Neugeborener & ઈ & 46,3 & ungefärbt \\
\hline 3 & $\begin{array}{l}\text { Bruchsack- } \\
\text { operation }\end{array}$ & - & む 58 & 52,0 & blau \\
\hline 4 & 192 & $\begin{array}{l}\text { Peritonitis d. Perforation eines } \\
\text { tuberkul. Coecalgeschwürs, } \\
\text { Lungentuberkulose }\end{array}$ & ㅇ 29 & $\begin{array}{l}49,7 \\
50,6\end{array}$ & $\begin{array}{l}\text { schmutzig } \\
\text { blaugrün } \\
\text { blau }\end{array}$ \\
\hline 5 & $926(1911)$ & $\begin{array}{c}\text { Arteriosklerose, Herzhypertrophie, } \\
\text { Cholelithiasis }\end{array}$ & ち 59 & 50,9 & , \\
\hline 6 & 383 & Peritonitis $\mathrm{n}$. Zangenentbindung & ㅇ 28 & 50,7 & , \\
\hline 7 & 246 & $\begin{array}{c}\text { Peritonitis nach Blinddarm- } \\
\text { operation }\end{array}$ & ㅇ 16 & 50,5 & > \\
\hline 8 & 139 & Adipositas univ., Gicht & む 62 & 50,8 & 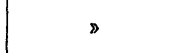 \\
\hline 9 & 393 & $\begin{array}{c}\text { Eklampsie. Parenchym. Degenerat. } \\
\text { der Nieren }\end{array}$ & ㅇ 32 & $\begin{array}{l}51,3 \\
50,0\end{array}$ & blaugrün \\
\hline 10 & 429 & $\begin{array}{c}\text { Peritonitis nach Exstirpation der } \\
\text { Genitalien w. Kystom }\end{array}$ & ㅇ 45 & $\begin{array}{l}50,7 \\
50,9\end{array}$ & blau \\
\hline 11 & 215 & $\begin{array}{l}\text { Eklampsie. Toxisch. Degen. d. } \\
\text { Leber u. Nieren. Compressio } \\
\text { cerebri durch Bluterguß }\end{array}$ & ㅇ 34 & 50,5 & 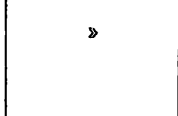 \\
\hline 12 & 972 (1911) & Atroph. Lebercirrhose, Nephritis & q 49 & วั1,0 & 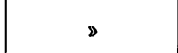 \\
\hline 13 & - & Ertrinkungstod (Suicid) & ㅇ 47 & $\begin{array}{l}50,8 \\
50,7\end{array}$ & D \\
\hline 14 & 472 & Akute Pankreasnekrose & q 32 & $\begin{array}{l}50,6 \\
50,2\end{array}$ & , \\
\hline 15 & 406 & $\begin{array}{l}\text { Kroupöse Pneumonie (vor } 2 \text { Jahren } \\
\text { Mammacarcinomexstirpation) }\end{array}$ & q57 & $\begin{array}{l}50,3 \\
50,0 \\
51,0\end{array}$ & $\begin{array}{c}\text { schmutzig blau } \\
\text { blau }\end{array}$ \\
\hline 16 & 391 & $\begin{array}{c}\text { Verblutung durch vorzeitige Lö- } \\
\text { sung der Placenta }\end{array}$ & ㅇ 41 & $\begin{array}{l}51,1 \\
50,5\end{array}$ & 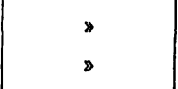 \\
\hline 17 & 240 & $\left|\begin{array}{c}\text { Erysipel d. Gesichts. Allgem. Sepsis } \\
\text { bei puerp. Endometritis }\end{array}\right|$ & q 35 & 51,0 & $D$ \\
\hline
\end{tabular}

1) Die Sekt.-Nrn. beziehen sich auf das Jahr 1912, falls nichts Gegenteiliges 
«Unverseifbarem* und "Gholesterin» bei Nichtcarcinom.

Darstellung Nr. 1.

\begin{tabular}{|c|c|c|c|c|c|c|c|}
\hline $\begin{array}{l}\text { Ort der } \\
\text { Entnahme }\end{array}$ & $\begin{array}{c}\text { Un- } \\
\text { verseif- } \\
\text { bares } \\
\text { in } \%\end{array}$ & $\begin{array}{c}\text { Un- } \\
\text { verseif- } \\
\text { bares } \\
\text { in \% } \\
\text { (Mittel- } \\
\text { zahl) }\end{array}$ & $\begin{array}{l}\text { Chole- } \\
\text { sterin- } \\
\text { gehalt } \\
\text { in } \%\end{array}$ & \begin{tabular}{|c|} 
Chole- \\
sterin- \\
gehalt \\
in $0 / 0$ \\
(Mittel- \\
zahl)
\end{tabular} & \begin{tabular}{|c|} 
Chole- \\
sterin- \\
gehalt \\
in \% des \\
Unverseif- \\
baren
\end{tabular} & $\begin{array}{c}\text { Phos- } \\
\text { phatid als } \\
\text { Lecithin } \\
\text { berechnet }\end{array}$ & $\begin{array}{c}\text { Be- } \\
\text { merkungen }\end{array}$ \\
\hline Frauenmilchfett & 0,3180 & 0,3180 & 0,0878 & 0,0878 & 27,6 & - & \\
\hline subcutan & 0,2128 & 0,2128 & 0,0922 & 0,0922 & 43,3 & - & \\
\hline , & 0,2204 & 0,2204 & 0,1390 & 0,1390 & 63,1 & - & \\
\hline Netz & 0,2730 & 0,2730 & 0,0769 & 0,0769 & 28,1 & - & \\
\hline Mesenterium & 0,3016 & & 0,1390 & & 46,0 & - & \\
\hline Netz & 0,3020 & 0,3018 & 0,1227 & 0,1308 & 40,6 & - & \\
\hline , & 0,3154 & 0,3154 & 0,1441 & 0,1441 & 45,6 & - & \\
\hline Oberschenkel & 0,3354 & 0,3354 & 0,0938 & 0,0938 & 28,2 & - & \\
\hline, & 0,3372 & 0,3372 & 0,0784 & 0,0784 & 23,2 & - & \\
\hline Bauchwand & 0,3448 & 0,3448 & 0,1606 & 0,1606 & 46,5 & $\begin{array}{l}\text { Keine Phos- } \\
\text { phorsaure } \\
\text { nachweisbar }\end{array}$ & $\begin{array}{l}\text { Zur Lecithin- } \\
\text { bestimmung } \\
\text { wurden } 150 \mathrm{~g}\end{array}$ \\
\hline $\begin{array}{l}\text { Oberschenkel } \\
\text { Mesenterium }\end{array}$ & $\begin{array}{l}0,2790 \\
0,4209\end{array}$ & 0,3499 & $\begin{array}{l}0,0563 \\
0,0938\end{array}$ & 0,0750 & $\begin{array}{l}20,1 \\
22,2\end{array}$ & - & \\
\hline $\begin{array}{l}\text { Oberschenkel } \\
\text { Mesenterium }\end{array}$ & $\begin{array}{l}0,3496 \\
0,3580\end{array}$ & 0,3538 & $\begin{array}{l}0,1014 \\
0,1506\end{array}$ & 0,1260 & $\begin{array}{l}29,0 \\
42,0\end{array}$ & - & \\
\hline, & 0,3562 & 0,3562 & 0,1627 & 0,1627 & 45,6 & 0,0775 & $\begin{array}{l}\text { Zur Lecithin- } \\
\text { bestimmung } \\
\text { wurden } 150 \mathrm{~g} \\
\text { Fett verbrannt. }\end{array}$ \\
\hline , & 0,3614 & 0,3614 & 0,1515 & 0,1515 & 41,9 & - & \\
\hline $\begin{array}{l}\text { Oberschenkel } \\
\text { Mesenterium }\end{array}$ & $\begin{array}{l}0,3202 \\
0,4096\end{array}$ & 0,3649 & $\begin{array}{l}0,1452 \\
0,1886\end{array}$ & 0,1669 & $\begin{array}{l}45,3 \\
46,0\end{array}$ & - & \\
\hline $\begin{array}{c}\text { Oberschenkel } \\
\text { Mamma }\end{array}$ & $\begin{array}{l}0,3616 \\
0,3977\end{array}$ & 0,3796 & $\begin{array}{l}0,1604 \\
0,1660\end{array}$ & 0,1632 & $\begin{array}{l}44,3 \\
41,7\end{array}$ & $\overline{-}$ & \\
\hline $\begin{array}{c}\text { Oberschenkel } \\
\text { Mesenterium } \\
\text { Mamma }\end{array}$ & $\begin{array}{l}0,3618 \\
0,4018 \\
0,3784\end{array}$ & 0,3807 & $\begin{array}{l}0,1566 \\
0,1444 \\
0,1431\end{array}$ & 0,1480 & $\begin{array}{l}43,0 \\
35,9 \\
37,8\end{array}$ & $\frac{-}{-}$ & \\
\hline $\begin{array}{l}\text { Oberschenkel } \\
\text { Mesenterium }\end{array}$ & $\begin{array}{l}0,3294 \\
0,4594\end{array}$ & 0,3944 & $\begin{array}{l}0,0805 \\
0,1892\end{array}$ & 0,1348 & $\begin{array}{l}24,7 \\
41,1\end{array}$ & - & \\
\hline Oberschenkel & 0,3970 & 0,3970 & 0,1497 & 0,1497 & 37,7 & - & \\
\hline
\end{tabular}

bemerkt ist. 
Tafel I.

\begin{tabular}{|c|c|c|c|c|c|}
\hline $\mathrm{Nr}$. & $\begin{array}{l}\text { Sektion } \\
\mathrm{Nr} .\end{array}$ & Diagnose & $\begin{array}{c}\text { Ge- } \\
\text { schlecht } \\
\text { und } \\
\text { Alter }\end{array}$ & $\mid \begin{array}{c}\text { Refrakto- } \\
\text { meter- } \\
\text { zahl } \\
\text { bei } 40^{\circ} \mathrm{C} .\end{array}$ & $\begin{array}{l}\text { Farbe des } \\
\text { Refrakto- } \\
\text { meterrandes }\end{array}$ \\
\hline 18 & 260 & Erysipel, Epilepsie & む 30 & $\begin{array}{l}51,4 \\
51,9\end{array}$ & blau \\
\hline 19 & $1086(1911)$ & Gehirngliom & 9 44 & 50,6 & > \\
\hline 20 & 248 & $\begin{array}{l}\text { Korsakoffsche Psychose. Pota- } \\
\text { torium. Sepsis bei Abszeß am } \\
\text { Oberschenkel }\end{array}$ & t 45 & $\begin{array}{l}51,0 \\
51,1\end{array}$ & , \\
\hline 21 & 183 & $\begin{array}{c}\text { Thrombose d. Herzohrs. Braune } \\
\text { Degenerat. des Myocards. } \\
\text { Adipositas univers. }\end{array}$ & む 75 & 51,2 & blaugrün \\
\hline 22 & 345 & Sarkom ulz. & q 82 & $\begin{array}{l}\check{5} 2,3 \\
51,3\end{array}$ & 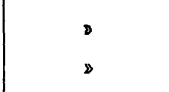 \\
\hline 23 & 195 & Arteriosklerose, Adipositas & 우 81 & 50,9 & blau \\
\hline 24 & 220 & $\begin{array}{l}\text { Embolie d. Lungenarterie. } \\
\text { Miliartuberkulose }\end{array}$ & ㅇ 34 & $\begin{array}{l}49,6 \\
50,0\end{array}$ & blaugrün \\
\hline 25 & 401 & Lebercirrhose, Miliartuberkulose & む 41 & $\begin{array}{l}49,9 \\
50,1\end{array}$ & $\begin{array}{l}\text { blau } \\
\text { blaugrün }\end{array}$ \\
\hline 26 & 198 & $\begin{array}{c}\text { Erysipel d. Gesichts. Cystopyelitis, } \\
\text { Bronchopneumonie }\end{array}$ & q 60 & 50,7 & $\begin{array}{l}\text { schmutzig } \\
\text { blaugrün }\end{array}$ \\
\hline 27 & 212 & Lungentuberkulose & \&5 & 50,8 & blaugrün \\
\hline 28 & 408 & Lungentuberkulose & む 43 & 49,8 & $\begin{array}{c}\text { schmutzig } \\
\text { blau }\end{array}$ \\
\hline 29 & $\begin{array}{c}1052 \\
(1911)\end{array}$ & $\begin{array}{l}\text { Erysipel, Arthritis urica. } \\
\text { Ulz. Dickdarmgeschwür }\end{array}$ & む 57 & 51,0 & blau \\
\hline 30 & $955(1911)$ & Diabetes, Arteriosklerose & む 63 & 50,8 & $\triangleright$ \\
\hline 31 & 396 & Lungentuberkulose & t 50 & $\begin{array}{l}50,4 \\
50,2\end{array}$ & blaugrün \\
\hline 32 & 367 & $\begin{array}{c}\text { Embolie n. Exstirp. d. Ovariums } \\
\text { wegen Cystoma ovarii }\end{array}$ & ㅇ 53 & $\begin{array}{l}51,1 \\
51,3\end{array}$ & $\begin{array}{c}\text { blau } \\
\text { blaugrün }\end{array}$ \\
\hline 33 & 284 & $\begin{array}{c}\text { Chron. Sepsis. } \\
\text { Hämolyt. Streptococcen }\end{array}$ & t 44 & $\begin{array}{l}51,1 \\
51,7\end{array}$ &, \\
\hline 34 & 427 & $\begin{array}{l}\text { Arteriosklerose, Lebercirrhose. } \\
\text { Gran. Atrophie d. Nieren. Oedem }\end{array}$ & む 58 & $\begin{array}{l}52,1 \\
51,7\end{array}$ & $\left\{\begin{array}{c}\text { blaugrün mit } \\
\text { stark gelbem } \\
\text { Rand }\end{array}\right.$ \\
\hline
\end{tabular}


Fortsetzing.

\begin{tabular}{|c|c|c|c|c|c|c|c|}
\hline $\begin{array}{l}\text { Ort der } \\
\text { Entnahme }\end{array}$ & $\begin{array}{c}\text { Un- } \\
\text { verseif- } \\
\text { bares } \\
\text { in } \%\end{array}$ & \begin{tabular}{|c|} 
Un- \\
verseif- \\
bares \\
in $\%$ \\
(Mittel- \\
zahl)
\end{tabular} & $\begin{array}{l}\text { Chole- } \\
\text { sterin- } \\
\text { gehalt } \\
\text { in } \% \text {. }\end{array}$ & $\begin{array}{l}\text { Chole- } \\
\text { sterin- } \\
\text { gehalt } \\
\text { in \% } \\
\text { (Mittel- } \\
\text { zahl) }\end{array}$ & $\begin{array}{l}\text { Chole- } \\
\text { sterin- } \\
\text { gehalt } \\
\text { in \% des } \\
\text { Unverseif- } \\
\text { baren }\end{array}$ & $\begin{array}{c}\text { Phos- } \\
\text { phatid als } \\
\text { Lecithin } \\
\text { berechnet }\end{array}$ & $\begin{array}{c}\mathrm{Be}- \\
\text { merkungen }\end{array}$ \\
\hline Oberschenkel & 0,2968 & & 0,0901 & & 30,3 & - & \\
\hline Mesenterium & 0,5036 & 0,4002 & 0,2320 & 0,1610 & 46,1 & - & \\
\hline Netz & 0,4080 & 0,4080 & 0,1320 & 0,1320 & 32,3 & - & \\
\hline Oberschenkel & 0,2690 & & 0,0806 & & 30,0 & - & \\
\hline Mesenterium & 0,5496 & 0,4093 & 0,2192 & 0,1499 & 39,8 & - & \\
\hline Oberschenkel & 0,4198 & 0,4198 & 0,1440 & 0,1440 & 34,3 & - & \\
\hline Oberschenkel & 0,4340 & & 0,1038 & & 23,9 & - & \\
\hline Mesenterium & 0,4486 & 0,4413 & 0,1414 & 0,1226 & 31,5 & - & \\
\hline , & 0,4654 & 0,4654 & 0,1940 & 0,1940 & 41,6 & - & \\
\hline Oberschenkel & 0,4408 & & 0,1646 & & 37,3 & - & \\
\hline Mesenterium & 0,5596 & 0,5002 & 0,2090 & 0,1868 & 37,3 & - & \\
\hline Oberschenkel & 0,3692 & & 0,1512 & & 40,9 & - & \\
\hline Mesenterium & 0,6420 & 0,5056 & 0,2792 & 0,2152 & 43,4 & - & \\
\hline , & $0, \check{5} 200$ & 0,5200 & 0,1400 & 0,1400 & 26,9 & - & \\
\hline $\begin{array}{l}\text { Mesenterium } \\
\text { und Netz }\end{array}$ & 0,5268 & 0,5268 & 0,2184 & 0,2184 & 41,4 & - & \\
\hline $\begin{array}{l}\text { Mesenterium } \\
\text { und subcutan }\end{array}$ & 0,5668 & 0,5668 & 0,1952 & 0,1952 & 34,4 & - & \\
\hline $\begin{array}{c}\text { Mesenterium } \\
\text { und Netz }\end{array}$ & $0, \bar{\jmath} 674$ & 0,5674 & 0,2770 & 0,2770 & 48,8 & - & \\
\hline $\begin{array}{l}\text { Mesenterium, } \\
\text { Netz, subcut. }\end{array}$ & 0,6662 & 0,6662 & 0,2812 & 0,2812 & 42,2 & - & \\
\hline Oberschenkel & 0,6040 & & 0,1610 & & 26,7 & - & \\
\hline Mesenterium & 0,7288 & 0,6664 & 0,2328 & 0,1971 & 31,9 & - & \\
\hline Oberschenkel & 0,4596 & 077713 & 0,0964 & 0,2842 & 20,9 & 一 & \\
\hline Mesenterium & 1,0830 & & 0,4720 & 0,2042 & 43,5 & - & \\
\hline $\begin{array}{c}\text { subcutan } \\
\text { (Bauchdecke) }\end{array}$ & 0,6952 & 1,1160 & 0,1419 & 0,3728 & 20,4 & - & \\
\hline Mesenterium & 1,5368 & 1,1100 & 0,6038 & & 39,2 & - & \\
\hline Oberschenkel & 0,9586 & & 0,2352 & 0,3078 & $24, \tilde{D}$ & - & \\
\hline Mesenterium & 1,4480 & 1,2033 & 0,3806 & 0,3078 & 26,2 & - & \\
\hline
\end{tabular}


Tafel Ia. Gehalt des menschlichen Depotfettes an

$\mathrm{Zu}$ graphischer

\begin{tabular}{|c|c|c|c|c|c|}
\hline $\begin{array}{l}\text { Lau- } \\
\text { fende } \\
\text { Nr. }\end{array}$ & $\begin{array}{c}\text { Sektion } \\
\mathrm{Nr} .\end{array}$ & Diagnose & $\begin{array}{l}\text { Ge- } \\
\text { schlecht } \\
\text { und } \\
\text { Alter }\end{array}$ & $\begin{array}{c}\text { Refrakto } \\
\text { meter- } \\
\text { zahl } \\
\text { bei } 40^{\circ} \mathrm{C} \text {. }\end{array}$ & $\begin{array}{c}\text { Farbe des } \\
\text { Refrakto- } \\
\text { meter- } \\
\text { randes }\end{array}$ \\
\hline 1 & Operation & Mammacarcinom & 우 33 & 49,8 & blau \\
\hline 2 & $1017(1911)$ & $\begin{array}{l}\text { Gastrostomie bei ulz. Uesophaguscarcinom } \\
\text { Pneumonie }\end{array}$ & t 61 & 50,4 & blaugrün \\
\hline 3 & Operation & Mammacarcinom & 우 46 & 50,6 & blau \\
\hline 4 & 376 & $\begin{array}{c}\text { Peritonitis nach Exstirpation des Uterus } \\
\text { wegen Carcinom }\end{array}$ & o 29 & 50,1 & blaugrün \\
\hline 5 & $\begin{array}{l}980 \\
(1911)\end{array}$ & $\begin{array}{l}\text { Peritonitis infolge Perforation eines } \\
\text { Rektumcarcinoms }\end{array}$ & ㅇ 55 & $\begin{array}{l}51,4 \\
51,3\end{array}$ & blau \\
\hline 6 & 193 & Lebercarcinom mit Metastasen & む 58 & $\begin{array}{l}50,5 \\
51,7\end{array}$ & $:$ \\
\hline 7 & 415 & $\begin{array}{c}\text { Med. Carcinom des Magens mit } \\
\text { Metastasen }\end{array}$ & 우 68 & $\begin{array}{l}50,5 \\
50,1\end{array}$ & blaugrün \\
\hline 8 & 422 & $\begin{array}{l}\text { Peritonitis nach Exstirpation der } \\
\text { Genital. wegen Carcinom }\end{array}$ & ㅇ 61 & $\begin{array}{l}52,2 \\
52,0 \\
51,5\end{array}$ & $\begin{array}{c}\text { blau } \\
\text { blaugrün } \\
\text { blaugrün mit } \\
\text { gelbem Rand }\end{array}$ \\
\hline 9 & 372 & $\begin{array}{c}\text { EmbolienachResektion des Rektums } \\
\text { wegen Carcinom (Adipositas) }\end{array}$ & 우 67 & $\begin{array}{l}51,0 \\
50,3\end{array}$ & blau \\
\hline 10 & 504 & $\begin{array}{l}\text { Embolie d. Lungenarterie, Mammacarcinom } \\
\text { und Carcinose der Pleurahöhle }\end{array}$ & .965 & 51,0 & , \\
\hline 11 & 986 (1911) & Magencarcinom m. Lebermetastasen & $q 62$ & 51,4 & $\begin{array}{l}\text { stark gelbgrüner } \\
\text { Rand }\end{array}$ \\
\hline 12 & 229 & Ulz. Oesophaguscarcinom & q40 & 52,0 & blaugrün \\
\hline 13 & 166 & $\begin{array}{l}\text { Peritonitis n. Exstirpation des Uterus } \\
\text { weg. Carcinom (Adipositas univers.) }\end{array}$ & \$40 & $\begin{array}{l}50,7 \\
50,6 \\
50,6\end{array}$ & : \\
\hline 14 & - & Rektumcarcinom & む 60 & 51,7 & , \\
\hline $1 \dot{0}$ & 380 & Nierencarcinom mit Metastasen & む 75 & $\begin{array}{l}51,4 \\
52,2 \\
52,0\end{array}$ & $\begin{array}{c}\text { blau } \\
\text { saun mit } \\
\text { blaugrün mit }\end{array}$ \\
\hline 16 & 359 & Jauch. Magencarcinom & \% 70 & $\begin{array}{l}51,0 \\
50,7\end{array}$ & $\begin{array}{l}\text { blaugrün } \\
\text { blaugrun mit } \\
\text { gelbem Rand }\end{array}$ \\
\hline 17 & 271 & $\begin{array}{l}\text { Mammacarcinom mit Leber- } \\
\text { metastasen }\end{array}$ & 우 55 & 50,0 & $\begin{array}{l}\text { blaugrün } \\
\text { blaagrïn mit } \\
\text { gelbem Rand }\end{array}$ \\
\hline 18 & 390 & Magencarcinom & む 60 & 51,4 & blaugrün \\
\hline 19 & 226 & $\begin{array}{c}\text { Peritonitis n. Exstirpation des Uterus } \\
\text { und Adnexe wegen Carcinom }\end{array}$ & † 48 & $\begin{array}{l}51,2 \\
51,0\end{array}$ & $\begin{array}{c}\text { schmutzig blau- } \\
\text { grüun } \\
\text { blaugrün }\end{array}$ \\
\hline 20 & 272 & Magencarcinom mit Metastasen & \& 47 & $\begin{array}{l}51,6 \\
50,0 \\
51,5\end{array}$ & $\begin{array}{l}\text { blaugrün mit } \\
\text { stark gelbem } \\
\text { Hand }\end{array}$ \\
\hline 21 & 157 & Ulz. Magencarcinom mit Metastasen & \&5 & 51,3 & $\begin{array}{l}\text { stark gelbgrüner } \\
\text { Rand }\end{array}$ \\
\hline 22 & 333 & $\begin{array}{l}\text { Ulz. fung. Pyloruscarcinom } \\
\text { Brought to you by }\end{array}$ & $\begin{array}{l}q 57 \\
\text { New } Y\end{array}$ & $\begin{array}{r}52,3 \\
51,1\end{array}$ & $\left\{\begin{array}{l}\text { blaugrün mit } \\
\text { gelbem Rand }\end{array}\right.$ \\
\hline
\end{tabular}


"Unverseifbarem» und "Cholesterin» bei Carcinom.

Darstellung Nr. 1.

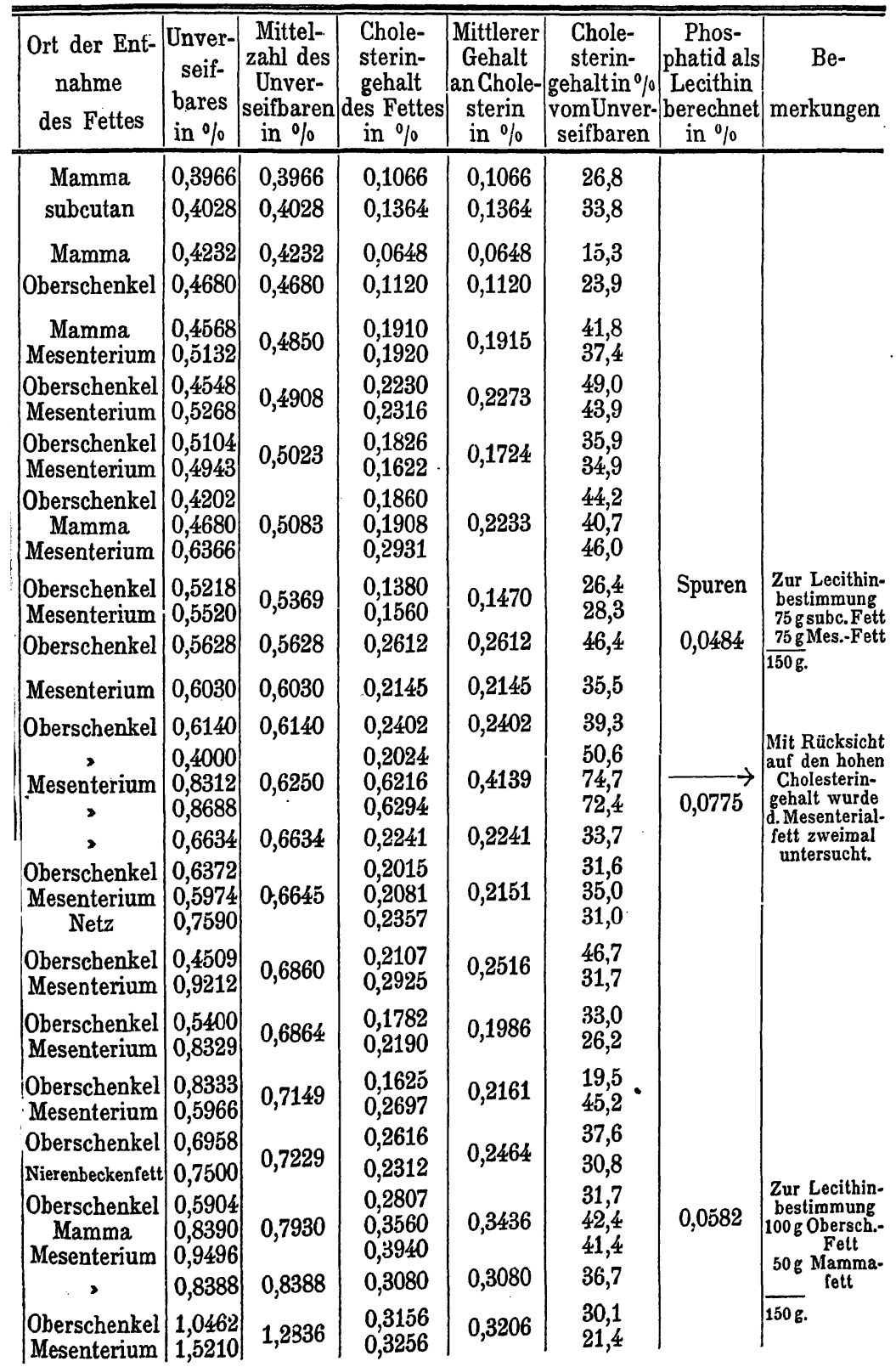


Graphische Darstellung №.. 2. Dazu Tafel 2 und 2a. Gehalt des menschlichen Untorhautfettes an, Unverseifbarer Substanz' und ,Cholesterin bei Carcinom und Nichtcarcinom.

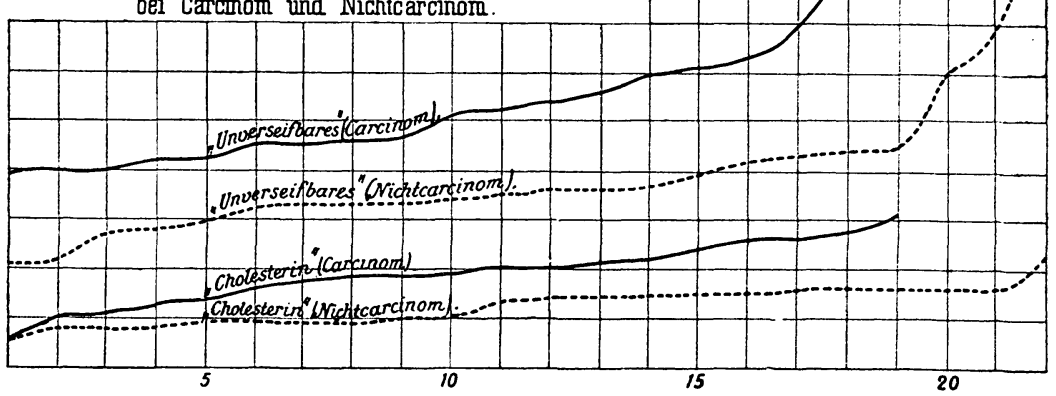

Graphische Darstellung №.3. Dazu Tafel 3 und $3^{\mathrm{a}}$ Gehalt des menschlichen Mesenterialfettes an. Unverseifbarer Substanz" und .Cholesterin " bei Carcinom and Nichtcarcinom.

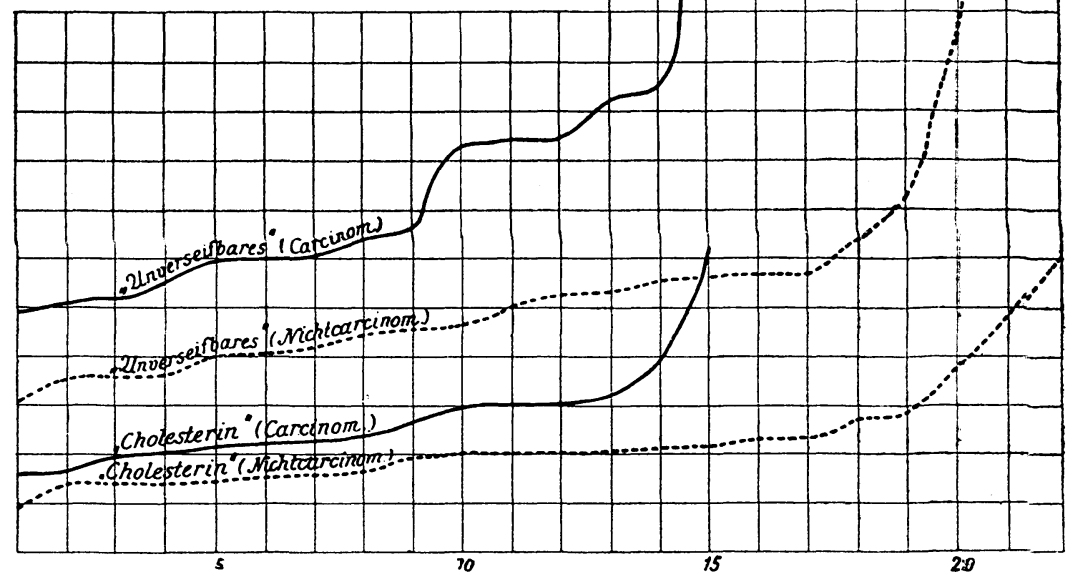


Das Cholesterin und seine Begleitsubstanzen.

Tafel II zu graphischer Darstellung Nr. 2.

Cholesteringehalt des menschliçhen Unterhautfettes bei Nichtcarcinom.

\begin{tabular}{|c|c|c|c|c|c|}
\hline Nr. & $\begin{array}{l}\text { Sektion } \\
\text { Nr. }\end{array}$ & Diagnose & $\begin{array}{l}\text { Chole- } \\
\text { sterin- } \\
\text { gehalt }\end{array}$ & $\begin{array}{l}\text { Unver- } \\
\text { seif- } \\
\text { bares }\end{array}$ & $\begin{array}{l}\text { Chole- } \\
\text { sterin in } \\
\% \text { des } \\
\text { Unver- } \\
\text { seif baren }\end{array}$ \\
\hline 1 & 393 & Eklampsie & 0,0561 & 0,2790 & 20,1 \\
\hline 2 & 246 & $\begin{array}{c}\text { Peritonitis nach Blindarm- } \\
\text { exstirpation }\end{array}$ & 0,0784 & 0,3372 & 23,1 \\
\hline 3 & 391 & $\begin{array}{c}\text { Verblutung durch vorzeitige } \\
\text { Lösung der Placenta }\end{array}$ & $0,0,805$ & 0,3294 & 24,7 \\
\hline 4 & 248 & $\begin{array}{l}\text { Korsakoffsche Psychose, } \\
\text { Abszeß des Oberschenkels }\end{array}$ & 0,0806 & 0,2690 & 30,0 \\
\hline $\mathbf{5}$ & 260 & Eryșipel, Epilepsie & 0,0901 & 0,2968 & 30,3 \\
\hline 6 & 6 & Bronchopneumonie & 0,0922 & 0,2128 & 43,3 \\
\hline 7 & 383 & $\begin{array}{c}\text { Peritonitis bei Zangen- } \\
\text { entbindung }\end{array}$ & 0,0938 & 0,3354 & $\underset{28,2}{2}$ \\
\hline 8 & 367 & $\begin{array}{c}\text { Embolie nach Exstirpation } \\
\text { des Ovariums wegen } \\
\text { Kystoms }\end{array}$ & 0,0964 & 0,4596 & 20,9 \\
\hline 9 & 429 & $\begin{array}{c}\text { Peritonitis n. Exstirpation } \\
\text { d. Genitalien }\end{array}$ & 0,1014 & 0,3496 & 29,0 \\
\hline 10 & 345 & Ulz. Sarkom & 0,1038 & 0,4340 & 23,9 \\
\hline 11 & - & Neugeborener & 0,1390 & 0,2204 & 63,1 \\
\hline 12 & 284 & $\begin{array}{l}\text { Chron. Sepsis, hämolyt. } \\
\text { Streptococcen }\end{array}$ & 0,1419 & 0,6952 & 20,4 \\
\hline 13 & 183 & Thrombose d. Herzohres & 0,1440 & 0,4198 & 34,3 \\
\hline 14 & $\begin{array}{c}\text { Gerichtlich } \\
\text { mediz. } \\
\text { Instit. }\end{array}$ & Ertrinkungstod (Suicid) & 0,1452 & 0,3202 & 45,3 \\
\hline 15 & 240 & Erysipel & 0,1497 & 0,3970 & 37,7 \\
\hline 16 & 401 & $\begin{array}{c}\text { Lebercirrhose, Miliar- } \\
\text { tuberkulose }\end{array}$ & 0,1512 & 0,3692 & 40,9 \\
\hline 17 & 406 & $\begin{array}{l}\text { Kroupöse Pneumonie, vor } \\
2 \text { Jahren Exstirpation der } \\
\text { Mamma wegen Carcinom }\end{array}$ & 0,1566 & 0,3618 & 43,0 \\
\hline 18 & 472 & Akute Pankreasnekrose & 0,1604 & 0,3616 & 44,3 \\
\hline 19 & 139 & Adipositas univ. Gicht & 0,1606 & 0,3448 & 46,5 \\
\hline 20. & 396 & Lungentuberkulose & 0,1615 & 0,6040 & 26,7 \\
\hline 21 & 220 & Miliartuberkulose & 0,1646 & 0,4408 & 37,3 \\
\hline 22 & 427 & $\begin{array}{c}\text { Arteriosklerose. } \\
\text { Gran.-Atrophie der Nieren } \\
\text { mit Oedẹm }\end{array}$ & 0,2350 & 0,9586 & 24,5 \\
\hline
\end{tabular}

Hoppe-Seyler's Zeitschrift f. physiol. Chemie. LXXX. 
Tafel IIa zu graphischer Darstellung Nr. 2.

Cholesteringehalt des menschlichen Unterhautfettes bei Carcinom.

\begin{tabular}{|c|c|c|c|c|c|}
\hline Nr. & $\begin{array}{l}\text { Sektion } \\
\text { Nr. }\end{array}$ & Diagnose & $\begin{array}{l}\text { Chole- } \\
\text { sterin- } \\
\text { gehalt } \\
\text { in } \%\end{array}$ & $\begin{array}{c}\text { Unver- } \\
\text { seif- } \\
\text { bares } \\
\text { in } \%\end{array}$ & $\begin{array}{l}\text { Chole- } \\
\text { sterin } \\
\text { in \% des } \\
\text { Unver- } \\
\text { seif baren }\end{array}$ \\
\hline 1 & Operation & Mammacarcinom & 0,0648 & 0,4232 & 15,3 \\
\hline 2 & , & , & 0,1066 & 0,3966 & 26,8 \\
\hline 3 & 376 & $\begin{array}{c}\text { Peritonitis nach } \\
\text { Exstirpation des Uterus } \\
\text { wegen Carcinom }\end{array}$ & 0,1120 & 0,4680 & 23,9 \\
\hline 4 & $1017(1911)$ & Oesophagus-Carcinom & 0,1364 & 0,4028 & 33,8 \\
\hline 5 & 372 & $\begin{array}{c}\text { Embolie nach Resektion } \\
\text { des Rektums wegen } \\
\text { Carcinom }\end{array}$ & 0,1380 & 0,5218 & 26,4 \\
\hline 6 & 390 & Magencarcinom & 0,1625 & 0,8388 & 19,5 \\
\hline 7 & 271 & Mammacarcinom & 0,1782 & 0,5400 & 33,0 \\
\hline 8 & 415 & $\begin{array}{c}\text { Med. Carcinom des } \\
\text { Magens mit Metastasen }\end{array}$ & 0,1826 & 0,5104 & 35,9 \\
\hline 9 & 422 & $\begin{array}{l}\text { Peritonitis nach Exstir- } \\
\text { pation der Genitalien } \\
\text { wegen Garcinom }\end{array}$ & 0,1860 & 0,4202 & 44,2 \\
\hline 10 & 980 & Rektumcarcinom & 0,1910 & 0,4568 & 41,8 \\
\hline 11 & 380 & Nierencarcinom & 0,2015 & 0,6372 & 31,6 \\
\hline 12 & 166 & $\begin{array}{l}\text { Peritonitis bei Uterus- } \\
\text { carcinom }\end{array}$ & 0,2024 & 0,4000 & 50,6 \\
\hline 13 & 359 & $\begin{array}{l}\text { Verjaucht. Magen- } \\
\text { carcinom }\end{array}$ & 0,2107 & 0,4509 & 46,7 \\
\hline 14 & 193 & $\begin{array}{l}\text { Lebercarcinom mit } \\
\text { Metastasen }\end{array}$ & 0,2230 & 0,4548 & 49,0 \\
\hline 15 & 229 & Oesophagus-Carcinom & 0,2402 & 0,6140 & 39,3 \\
\hline 16 & 504 & Mammacarcinom & 0,2612 & 0,5628 & 46,4 \\
\hline 17 & 226 & $\begin{array}{l}\text { Peritonitis nach Exstir- } \\
\text { pation des Uterus und } \\
\text { Âdnexe wegen Carcinom }\end{array}$ & 0,2616 & 0,6958 & 37,6 \\
\hline 18 & 272 & Magencarcinom & 0,2807 & 0,5904 & 31,7 \\
\hline 19 & 333 & $\begin{array}{l}\text { Ulz. fung. Pylorus- } \\
\text { carcinom }\end{array}$ & 0,3156 & 1,0462 & 30,1 \\
\hline
\end{tabular}


Das Cholesterin und seine Begleitsubstanzen.

Tafel III zu graphischer Darstellung Nr. 3.

Cholesteringehalt des menschlichen Mesenterialfettes bei Nichtcarcinom.

\begin{tabular}{|c|c|c|c|c|c|}
\hline Nr. & $\begin{array}{c}\text { Sektion } \\
\text { Nr. }\end{array}$ & Diagnose & $\begin{array}{c}\text { Cholesterin- } \\
\text { gehalt } \\
\text { in } \%\end{array}$ & \begin{tabular}{|} 
Unver- \\
seifbares \\
in $\%$
\end{tabular} & $\begin{array}{l}\text { Cholesterin- } \\
\text { gehalt des } \\
\text { Unverseif- } \\
\text { baren } \\
\text { in } \%\end{array}$ \\
\hline 1 & 391 & Eklampsie & 0,0938 & 0,4209 & 22,2 \\
\hline 2 & 192 & $\begin{array}{c}\text { Peritonitis d.Perforation bei } \\
\text { tuberkul. Coecalgeschwür }\end{array}$ & 0,1390 & 0,3016 & 46,0 \\
\hline 3 & 198 & $\begin{array}{c}\text { Erysipel des Gesichts, Cysto- } \\
\text { pyelitis }\end{array}$ & 0,1400 & 0,5200 & 26,9 \\
\hline 4 & 345 & Sarkom ulz. & 0,1414 & 0,4486 & 31,5 \\
\hline 5 & 406 & $\begin{array}{c}\text { Krup.Pneumonie vor } 2 \text { Jahr., } \\
\text { Exstirpation der Mamma } \\
\text { wegen Carcinom }\end{array}$ & 0,144 & 0,4018 & 35,9 \\
\hline 6 & 429 & $\begin{array}{c}\text { Peritonitis n. Exstirpation } \\
\text { der Genitalien }\end{array}$ & 0,1506 & 0,3580 & 42,0 \\
\hline 7 & 972 & Lebercirrhose & 0,1515 & 0,3614 & 41,9 \\
\hline 8 & 215 & Eklampsie & 0,1627 & 0,3562 & 45,6 \\
\hline 9 & $\begin{array}{c}\text { Gericht. } \\
\text { med. Inst. }\end{array}$ & Ertrinkungstod, Suicid & 0,1886 & 0,4096 & 46,0 \\
\hline 10 & 391 & Vorzeit. Lösung d. Placenta & 0,1892 & 0,4594 & 41,1 \\
\hline 11 & 195 & Arteriosklerose, Adipos. & 0,1940 & 0,4654 & 41,6 \\
\hline 12 & 408 & Lungentuberkulose & 0,1952 & 0,5668 & 34,4 \\
\hline 13 & 220 & Miliartuberkulose & 0,2090 & 0,55596 & 37,3 \\
\hline 14 & 212 & Lungentuberkulose & 0,2184 & 0,5268 & 41,4 \\
\hline 15 & 248 & $\begin{array}{c}\text { Korsak of sche Psychose } \\
\text { Oberschenkelabszeß }\end{array}$ & 0,2190 & 0,5496 & 39,8 \\
\hline 16 & 260 & Erysipel, Epilepsie & 0,2320 & 0,5036 & 46,1 \\
\hline 17 & 396 & Lungentuberkulose & 0,2328 & 0,7288 & 31,9 \\
\hline 18 & $\begin{array}{c}1052 \\
(1911)\end{array}$ & $\begin{array}{c}\text { Erysipel, ulz. Dickdarm- } \\
\text { geschwür }\end{array}$ & 0,2770 & 0,5674 & 48,8 \\
\hline 19 & 401 & $\begin{array}{c}\text { Lebercirrhose, Miliar- } \\
\text { tuberkulose }\end{array}$ & 0,2792 & 0,6420 & 43,4 \\
\hline 20 & 427 & $\begin{array}{c}\text { Arteriosklerose, Gran.-Atro- } \\
\text { phie der Nieren, Oedem }\end{array}$ & 0,3806 & 1,4480 & 26,2 \\
\hline 21 & 367 & $\begin{array}{c}\text { Embolie n. Exstirpation des } \\
\text { Ovariums }\end{array}$ & 0,4720 & 1,0830 & 43,5 \\
\hline 22 & 284 & $\left|\begin{array}{c}\text { Chron. Sepsis, Hämolytische } \\
\text { Streptococcen }\end{array}\right|$ & 0,6038 & 1,5368 & 39,2 \\
\hline
\end{tabular}


Tafel III a zu graphischer Darstellung Nr. 3.

Cholesteringehalt des menschlichen Mesenterialfettes bei Carcinom.

\begin{tabular}{|c|c|c|c|c|c|}
\hline Nr. & $\begin{array}{l}\text { Sektion } \\
\mathrm{Nr} .\end{array}$ & Diagnose & $\begin{array}{l}\text { Chole- } \\
\text { sterin- } \\
\text { gehalt } \\
\text { in } \%\end{array}$ & $\begin{array}{l}\text { Unver- } \\
\text { seif- } \\
\text { bares } \\
\text { in } \%\end{array}$ & $\begin{array}{c}\text { Cholestern- } \\
\text { gehalt } \\
\text { des Unver- } \\
\text { seifbaren } \\
\text { in } \%\end{array}$ \\
\hline 1 & 372 & $\begin{array}{l}\text { Embolie nach Resektion des } \\
\text { Rektums wegen Carcinom }\end{array}$ & 0,1560 & 0,5520 & 38,3 \\
\hline 2 & 415 & $\begin{array}{l}\text { Med. Magencarcinom mit } \\
\text { Metastasen }\end{array}$ & 0,1622 & 0,4943 & 34,9 \\
\hline 3 & 9.80 & Rektumcarcinom & 0,1920 & 0,5132 & 37,4 \\
\hline 4 & 380 & $\begin{array}{c}\text { Nierencarcinom mit Meta- } \\
\text { stasen }\end{array}$ & 0,2081 & 0,5974 & 35,0 \\
\hline 5 & 986 & Magencarcinom & 0,2145 & 0,6030 & $3 \check{5}, 5$ \\
\hline 6 & 271 & $\begin{array}{c}\text { Mammacarcinom mit Meta- } \\
\text { stasen }\end{array}$ & 0,2190 & 0,8329 & 26,2 \\
\hline 7 & - & Rektumcarcinom & 0,2241 & 0,6634 & 33,7 \\
\hline 8 & 193 & $\begin{array}{l}\text { Lebercarcinom mit Meta- } \\
\text { stasen }\end{array}$ & 0,2316 & 0,5268 & 43,9 \\
\hline 9 & 390 & Magencarcinom & 0,2697 & 0,5966 & 45,2 \\
\hline 10 & 359 & Jauch. Magencarcinom & 0,2925 & 0,9212 & 31,7 \\
\hline 11 & 422 & $\begin{array}{c}\text { Peritonitis n. Exstirpation } \\
\text { der Genitalien wegen } \\
\text { Carcinom }\end{array}$ & 0,2931 & 0,6366 & 46,0 \\
\hline 12 & 157 & Ulz. Magencarcinom & 0,3080 & 0,8388 & 36,7 \\
\hline 13 & 333 & Ulz. fung. Pyloruscarcinom & 0,3256 & 1,5210 & 21,4 \\
\hline 14 & 272 & Magencarcinom & 0,3940 & $0,9.496$ & 41,4 \\
\hline 15 & 166 & $\begin{array}{l}\text { Peritonitis n. Totalexstirpa- } \\
\text { tion des Uterus wegen Carci- } \\
\text { nom. Adipositas univers. }\end{array}$ & 0,6294 & 0,8688 & 72,4 \\
\hline
\end{tabular}

In den Tafeln, wie bei der Herstellung der Kurven, wurden die Fälle nach dem Prozentgehalt an Unverseifbarem, bezw. Cholesterin geordnet. Daraus ergibt sich, daß die untere Cholesterinkurve nicht von Fall zu Fall genau der darüber befindlichen Kurve des Unverseifbaren entspricht, obwohl im allgemeinen bei hohem Gehalt an Unverseifbarem auch das Cholesterin vermehrt ist. Zur Herstellung der Kurven sei erwähnt, daß die in den Tabellen aufgeführten Fälle in Abständen von je $10 \mathrm{zu} 10 \mathrm{~mm}$ auf der Abscissenachse aufgetragen und der 
Prozentgehalt an Unverseif barem bezw. Cholesterin multipliziert mit 100 in Millimeter auf der Ordinate abgemessen wurde. Enthält z. B. ein Fett $0,2128 \%$ Unverseif bares, so befindet sich der Schnittpunkt der Kurve 21,28 mm von der Abscissenachse nach aufwärts. Bei der Reproduktion wurden die so erhaltenen Kurven um die Hälfte linear verkleinert. (Frauenmilchfett wurde nicht in die Kurve aufgenommen.)

Aus den Tabellen und graphischen Darstellungen geht zunächst hervor, daß das Unterhautfett in den meisten Fällen weniger Unverseifbares und weniger Cholesterin enthält wie das Mesenterialfett.

Fette bei akuten Infektionskrankheiten (Peritonitis, Pneumonie), operativ gewonnenes Fett, Fett bei Unglücksfällen, Eklampsie zeigen die geringsten Werte an gelösten Lipoidsubstanzen. Etwas Erhöhung ist schon bei Erysipel, Arteriosklerose, Sarkom bemerkbar. Ziemlich beträchtlich steigt der Gehalt bei Carcinom, Tuberkulose, Diabetes, Kystoma ovarii, chrotiischer Sepsis, Arteriosklerose mit Granularatrophie der Nieren mit Ödem.

\begin{tabular}{|c|c|c|c|c|c|c|}
\hline \multirow[b]{2}{*}{ bei } & \multicolumn{2}{|c|}{$\begin{array}{l}\text { Mittlerer Prozent- } \\
\text { gehalt des mensch- } \\
\text { lichen Mesenterial- } \\
\text { und Unterhautfettes } \\
\text { an }\end{array}$} & \multicolumn{2}{|c|}{$\begin{array}{c}\text { Mittlerer } \\
\text { Prozentgehalt } \\
\text { des menschlichen } \\
\text { Unterhautfettes } \\
\text { an }\end{array}$} & \multicolumn{2}{|c|}{$\begin{array}{c}\text { Mittlerer } \\
\text { Prozentgehalt } \\
\text { des menschlichen } \\
\text { Mesenterialfettes } \\
\text { an }\end{array}$} \\
\hline & $\begin{array}{l}\text { Unver- } \\
\text { seifbarem }\end{array}$ & & $\begin{array}{l}\text { Unver- } \\
\text { seif barem }\end{array}$ & & $\begin{array}{c}\text { Unver- } \\
\text { seifbarem }\end{array}$ & \\
\hline $\begin{array}{l}\text { Nicht- } \\
\text { carcinom }\end{array}$ & $\begin{array}{c}0,8301 \\
\text { Taf. I } \\
\left.(1-16)^{1}\right)\end{array}$ & $\begin{array}{c}0,1877 \\
\text { Taf. I } \\
\left.(1-16)^{1}\right)\end{array}$ & $\begin{array}{c}0,8090 \\
\text { Taf. II } \\
\left.(1-13)^{i}\right)\end{array}$ & $\begin{array}{c}0,1108 \\
\text { Taf. II } \\
\left.(1-13)^{1}\right)\end{array}$ & $\begin{array}{c}0,4064 \\
\text { Taf. III } \\
\left.(1-11)^{2}\right) \\
\text { exkl. Nr. } 4\end{array}$ & $\begin{array}{c}0,1658 \\
\text { Taf. III } \\
\left.(1-11)^{8}\right) \\
\text { exkl. Nr. } 4\end{array}$ \\
\hline Cárcinom & $\begin{array}{c}0,6212 \\
\text { Tăf. Iá } \\
\text { (22 Fälle) }\end{array}$ & $\begin{array}{c}0,2198 \\
\text { Tâf. I } \\
(22 \text { Fälle) }\end{array}$ & $\begin{array}{c}0,5489 \\
\text { Taf. II à } \\
(19 \text { Fälle) }\end{array}$ & $\begin{array}{c}0,1923 \\
\text { Tá. II a } \\
\text { (19 Fälle) }\end{array}$ & $\begin{array}{c}\text { 0,7410 } \\
\text { Taf. Iila } \\
\text { (15 Fäle) }\end{array}$ & $\begin{array}{c}0,2746 \\
\text { Taf. IIIá } \\
(15 \text { Fâllè) }\end{array}$ \\
\hline $\begin{array}{l}\text { Tuber- } \\
\text { kulose }\end{array}$ & $\begin{array}{c}\text { 0,5ั532 } \\
\text { Tàf. I } \\
\text { (5 Fälle) }\end{array}$ & $\begin{array}{c}\text { 0,2025 } \\
\text { Taf. I } \\
\text { (5 Fälle) }\end{array}$ & $\begin{array}{c}\mathbf{0}, \mathbf{4 7 1 3} \\
\text { Taf. I } \\
\text { (3 Fälle) }\end{array}$ & $\begin{array}{c}0,1591 \\
\text { Täf: I } \\
\text { (3 Fälle) }\end{array}$ & $\begin{array}{c}0,6434 \\
\text { Taf. I } \\
\text { (3 Fälle) }\end{array}$ & $\begin{array}{c}\text { 6,2403 } \\
\text { Tăf. I } \\
\text { (3 Fälle) }\end{array}$ \\
\hline
\end{tabular}

1) Dem Prozentgehalt ân Unverseifbaàein nach geórdnët.

2) Dem Prožžnţ̧ểalt an Cholèstèrin nách geordnet. 
Der Übersichtlichkeit halber habe ich Mittelzahlen aus meinen Resultaten in vorstehender Tabelle nochmals zusammen. gestellt.

Begleitsubstanzen des Cholesterins im menschlicher.

$$
\text { Depotfett. }
$$

Die Begleitsubstanz des Cholesterins ist nicht durch Digitonin fällbar. Sie bildet den Hauptbestandteil der im: Fette gelösten Lipoidstoffe und zwar enthält

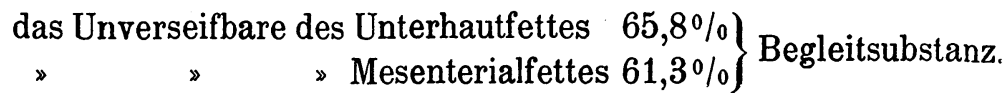

Zur Darstellung verfährt man wie folgt:

Die unverseifbare Substanz des menschlichen Fettes, wie sie nach Bömers Vorschrift (loc. cit.) erhalten wird, löst man in Alkohol und läßt 24 Stunden stehen. Es scheiden sich dadurch sehr geringe Mengen einer Substanz ab, die man $a b-$ filtriert. Das alkoholische Filtrat konzentriert man, bis sich beim Abkühlen der größte Teil des Cholesterins in krystallisierter Form abscheidet. Die Mutterlauge vom abgeschiedenen Cholesterin verdünnt man wiederum mit Alkohol und fällt daraus durch 1\% ige Digitoninlösung ${ }^{1}$ ) in $90 \%$ igem Alkohol den Rest des Cholesterins. Durch Probeentnahme überzeugt man sich, ob das Cholesterin vollkommen gefällt ist. Nach mehrstündigem Stehen saugt man das Digitonin-Cholesterid ab und dampft das alkoholische Filtrat zur Trockne ein. Aus dem getrockneten Rückstand extrahiert man die Begleitsubstanz mit Äther oder Petroläther. Im Rückstand bleibt allenfallsiges Digitonin-Cholesterid und überschüssiges Digitonin.

Nach dem Verdampfen des Äthers und Trocknen des Rückstands hinterbleibt eine wachsartige von $25-32^{\circ}$ schmelzende, Substanz, die analog dem Cholesterin die Eigenschaft besitzt, Wasser in sich aufzunehmen, bezw. mit viel Wasser eine vollkommen milchige Emulsion zu geben. Unter dem Mikroskop, besteht diese Emulsion aus zahllosen größeren und kleinerem

1) Das hiezu benützte Digitonin wurde vorher mit Äther extrahiert, um allenfallsige ätherlösliche Verunreinigungen fortzulösen. 
Bläschen. Myelinfiguren liefert die Begleitsubstanz nicht, sondern sie teilt sich, wie erwähnt, in kleine Bläschen auf. Aus der wässerigen Emulsion ist die Substanz schwer mit Äther extrahierbar, weil die Trennung der beiden Schichten erst nach tagelangem Stehen erfolgt.

Die Begleitsubstanz ist in den meisten Lösungsmitteln, wie Alkohol, Äther, Petroläther, Eisessig, leichtlöslich und konnte nicht zur Krystallisation gebracht werden. Gegen Alkali ist sie wenig beständig. Kocht man sie mit Natriumalkoholat oder alkoholischer Kalilauge im Wasserbad, so scheidet sich bei Verdünnung mit Wasser eine braune harzartige Masse aus. Aus dem alkalischen Filtrat des braunen Harzes fällt Salzsäure anscheinend eine Fettsäure. Es liegt daher der Gedanke nahe, daß die Begleitsubstanz der Fettsäureester eines cholesterinartigen, hochmolekularen Alkohols ist. Jedenfalls liegt ein Stoff vor, der für die chemische Untersuchung recht unerquickliche Eigenschaften besitzt.

In Nachfolgendem habe ich einige Reaktionen des Cholesterins denjenigen der Begleitsubstanz vergleichend gegenübergestellt:

\begin{tabular}{|c|c|c|}
\hline Art des Reagenzes & Cholesterin & Begleitsubstanz \\
\hline $\begin{array}{c}\text { Salkowskische Reagens. } \\
\text { Chloroform, konzentrierte } \\
\text { Schwefelsăure. }\end{array}$ & $\begin{array}{c}\text { Chloroform: blutrot } \\
\text { Schwefelsaure. Schwach } \\
\text { grüne Fluorescenz }\end{array}$ & $\begin{array}{c}\text { Chloroform: Schwach } \\
\text { gelb } \\
\text { Schwefelsäure: Braunrot }\end{array}$ \\
\hline $\begin{array}{l}\text { Li ebermannsche Reakt. } \\
\text { Chloroform, Essigsüure- } \\
\text { anhydrid konzentrierte } \\
\text { Schwefelsäure. }\end{array}$ & Rosarot dann blau & Braunrot \\
\hline $\begin{array}{c}\text { Golodetzsche Reaktion. } \\
\text { Schwefelsäure- } \\
\text { Formaldehyd. }\end{array}$ & Schwarzbraun & Braunrot \\
\hline Bromwasser in Eisessig. & Krystalle von Dibromid & Keine Krystalle \\
\hline $\begin{array}{l}\text { Essigsäureanhydrid } \\
\text { anhaltendes Kochen. }\end{array}$ & Esterbildung & Keine Veränderung \\
\hline $\begin{array}{l}\text { Digitoninlösung } \\
\text { in } 90 \% \text { Alkohol. }\end{array}$ & $\begin{array}{c}\text { Niederschlag von } \\
\text { Digitonin-Cholesterid }\end{array}$ & Kein Niederschlag \\
\hline
\end{tabular}


Die Begleitsubstanz ist demnach chemisch ziemlich indifferent, ihre physiologische Bedeutung dürfte daher wahrscheinlich mehr in ihrem physikalischen Verhalten zu suchen sein.

Die oben auf Seite 404 erwähnte, in Alkohol unlösliche Substanz scheidet sich beim Erkalten der warmen Spirituslösung des Unverseifbaren schon lange vor dem Cholesterin als leichte Trübung aus. Die bei zahlreichen Versuchen gesammelte geringe Menge wurde nochmals aus heißem Alkohol krystallisiert. Man erhält so einen weißen, bei $62-63^{\circ} \mathrm{G}$. schmelzenden Körper, der weder Cholesterin- noch Cholesterinesterreaktionen zeigt und bei ca. 50 facher Vergrößerung unter dem Mikroskop zuweilen in ganz feinen Nadeln, manchmal in derberen, konzentrisch gruppierten oder tannenzweigähnlichen Gebilden erscheint.

Gegen alkoholische Kalilauge ist die Substanz auch beim Kochen beständig und unterscheidet sich dadurch von dem Tristearin, ${ }^{1}$ ) das selbst bei wiederholter Krystallisation einen ähnlichen Schmelzpunkt zeigt.

Auf phosphorhaltige Lipoide (Lecithin) wurden 6 Fette untersucht. Drei Carcinomfette und ein Fett bei Nichtcarcinom zeigten wägbare Mengen von Phosphorsäure, ein Carcinomfett und ein Fett bei Nichtcarcinom enthielten keine oder doch nur Spuren von Phosphorsäure. Zum Vergleiche herangezogenes Kuhbutterfett (2 Proben) enthielten 0,0775\% Phosphatid (als Lecithin berechnet).

Um bei der Bestimmung der Phosphorsäure, die mit der Verseifung eingeführten großen Alkali- bezw. Salzmengen auszuschließen und die damit verbundene Gefahr der Kieselsäure-

1) Das hartnäckig verunreinigte bei ca. $62^{\circ}$ schmelzende Tristearin läbt sich aus den bei gewöhnlicher Temperatur aus dem menschlichen Fette sich abscheidenden festen Bestandteilen isolieren. Man filtriert zunächst bei Zimmertemperatur durch ein Faltenfilter und läßt den öligen Teil gut abtropfen, den Filterrückstand löst man in Äther und fällt mit der 2 fachen Menge, bezw. so viel Alkohol, bis ein Niederschläg entsteht. Wiederholt man nach der Filtration des Niederschlags diesen Prozeß, so erhält man schließlich ein Produkt; das aüs Alkohol in Nadeln krystallisiert und der Hauptsache nach aus Tristearin besteht. 
éinfuhr zu vermeidén, habe ich die Fette langsam mit Hilfe eines dünnen Dochtes aus zusammengedrehten Streifen aschenfreien Filtrierpapières in zwei Platinschalen (nach Art eines Nachtlichtes) verbrannt. Die Filtrierpapierdochte waren an Platindrăhten befestigt. Auf diese Weise gelingt es unter Verwendung von je 2 Flämmchen in $8-10$ Stunden die zur Analyse mindestens erforderlichen $150 \mathrm{~g}$ Fett $\mathrm{zu}$ verbrennen, dabei ist darauf zu achten, daß keine stark rußende Flamme entsteht. Zum Schluß wurden auch die Dochte verascht und der mit verdünnter heißer Salpetersäure extrahierte Rückstand mit molybdänsaurem Ammon gefällt. Die Phosphorsäure wurde in Form von pyrophosphorsaurer Magnesia zur Wägung gebracht. Zur Berechnung auf Lecithin multipliziert man letztere mit der Zahl 7,27.

Es kamen bei diesen Analysen nur 1-2 mg pyrophosphorsaurer Magnesia zur Wägung, d. h. Mengen, welche schon nahe der analytischen Fehlergrenze sind.

Fassen wir die Resultate dieser Untersuchung zusammen, so kommen wir $\mathrm{zu}$ folgenden Schlußfolgerungen:

1. Die Menge der sogenannten unverseifbaren Substanzen und damit auch des Cholesterins ist im menschlichen Depotfett bei Carcinom stark vermehrt.

2. Diese Anhäufung der Cholesterinsubstanzen, die äußerlich durch Gelbfärbung gekennzeichnet ist, erwies sich als für Carcinom nicht spezifisch. Sie findet sich auch bei chronisch verlaufenden Infektionskrankheiten wie Tuberkulose, chronischer Sepsis und bei Stoffwechselkrankheiten wie Diabetes, sowie im gelben Fette alter Personen.

3. Der Zuwachs an diesen unverseifbaren hochmolekularen Alkoholsubstanzen erfolgt meist zuerst und am reichlichsten im Mesenterialfett. Erst später beladet sich auch das Unterhautfett.

4. Verschiedene Anzeichen, z. B. das Fehlen der Cholesterinsubstanzen in den Nebennieren beim Carcinom usw., die Lipoidämie beim Coma diabeticum sprechen dafür, daß diese Erscheinungen vielleicht nicht lediglich auf Zellzerfall zurückzuführen sind, 
bezw. daß diesen Stoffen gleichzeitig eine entgiftende Wirkung in dem kranken Organismus zukommen dürfte. Weitere Untersuchungen müssen lehren, ob die säurebindende Eigenschaft des Cholesterins gleichzeitig eine Abwehrmaßregel des Organismus gegen die schädliche Wirkung organischer Fettsäuren darstellt.

5. Ein Nebenbefund der Untersuchung ist die Isolierung anderer Körper, insbesondere einer wachsartigen Begleitsubstanz des Cholesterins. Diese findet sich im unverseifbaren Teil des Fettes in größerer Menge als das Cholesterin (ungefähres Verhältnis 2:1). 